\title{
Plant-Expressed Recombinant Mountain Cedar Allergen Jun a 1 Is Allergenic and Has Limited Pectate Lyase Activity
}

\author{
Zun Liu ${ }^{a}$ Shikha Bhattacharyya ${ }^{a}$ Bo Ning ${ }^{b}$ Terumi Midoro-Horiuti ${ }^{b}$ \\ Edmund W. Czerwinski $^{b}$ Randall M. Goldblum ${ }^{b}$ Andrew Mort ${ }^{c}$ \\ Christopher M. Kearney ${ }^{\text {a }}$ \\ aDepartment of Biology, Baylor University, Waco, Tex., ${ }^{\text {b }}$ Department of Pediatrics, University of Texas \\ Medical Branch, Galveston, Tex., and 'Biochemistry and Molecular Biology, Oklahoma State University, \\ Stillwater, Okla., USA
}

\section{Key Words}

Jun a 1 - Juniperus ashei $\cdot$ Nicotiana benthamiana

Agrobacterium $\cdot$ Immunoglobulin E $\cdot$ Apoplast $\cdot$ Necrosis

\begin{abstract}
Background: Mountain cedar (Juniperus ashei) pollen commonly causes a winter time allergic rhinitis in the central USA. Jun a 1 is the dominant allergenic protein, but biologically active recombinant Jun a 1 has not been successfully expressed, despite numerous attempts with several expression systems. Method: Jun a 1 cDNA was inserted into a tobacco mosaic virus vector and transferred to Agrobacterium tumefaciens. Bacteria were syringe-inoculated into leaves of Nicotiana benthamiana (agroinoculation). The interstitial (apoplastic) fluid containing Jun a 1 was isolated. The recombinant protein was analyzed by SDS-PAGE, N-terminal sequencing and MALDI-TOF to confirm identity. Immunogenicity was examined with IgE from allergic patient's sera, mouse monoclonal anti-Jun a 1 antibodies, IgE-binding inhibition and by degranulation of RBL SX-38 cells sensitized with sera from allergic patients. Pectate lyase activity was assayed by capillary zone electrophoresis and mass spectrometry analysis. Results: Recombinant Jun a 1 was recovered in
\end{abstract}

good quantity (100 $\mu \mathrm{g} / \mathrm{g}$ leaf material), was confirmed as Jun a 1, bound IgE from sera from cedar hypersensitive patients and inhibited IgE binding to native Jun a 1. Jun a 1 mutants were created and their pectate lyase activity quantified. For the first time, Jun a 1 pectate lyase activity was demonstrated, which may explain the necrosis seen on host plants, which was similar to that of control plants expressing banana pectate lyase. Conclusions: A means of producing recombinant Jun a 1 is now available for structure/function studies and potentially for diagnostic and therapeutic uses.

Copyright $\odot 2010$ S. Karger AG, Basel

\section{Introduction}

Cedar pollen from the mountain cedar Juniperus ashei is one of the major causes of seasonal allergic rhinitis in the central USA [1]. Jun a 1, the dominant allergenic protein of this pollen, has been well characterized, beginning with its initial isolation by the authors (T.M.-H. and R.M.G.) in 1999 [2]. After cloning the cDNA of Jun a 1 in 1999 , its sequence indicated that it belongs to a family of pectate lyase enzymes [3]. Linear IgE epitopes of Jun a 1 were then identified using synthetic overlapping peptides

\section{KARGER}

(C) 2010 S. Karger AG, Basel

Fax +41613061234 E-Mail karger@karger.ch www.karger.com
Correspondence to: Dr. Christopher M. Kearney

Department of Biology, Baylor University

One Bear Place \#7388

Waco, TX 76798 (USA)

Tel. +1 254710 2131, Fax +1 254710 2969, E-Mail chris_kearney@baylor.edu 
and serum IgE from mountain cedar hypersensitive patients [4]. Finally, the crystal structure of Jun a 1 was determined from native protein and the previously identified linear epitopes were mapped to surface structures in 2005 by the authors [5].

Despite these advances, recombinant expression of soluble and folded Jun a 1 has been difficult to achieve, precluding detailed molecular studies of its allergenic or enzymatic mechanisms. Attempts at expressing Jun a 1 in bacteria, yeast and baculovirus systems has not yielded a tractable system [unpubl. observation, T.M.-H. and R.M.G.]. We reasoned that this plant allergen would be most easily expressed in a plant expression system. In fact, heterologous recombinant allergens have been efficiently produced in plants via tobacco mosaic virus (TMV) vectors in our laboratory [6] and by others [7-9].

A heterologous expression system would allow the molecular study of the potential pectate lyase activity of Jun a 1 , which is structurally similar to bacterial pectate and pectin lyases [5]. Plant pectate lyases promote germination by pollen grains and the ripening (softening) of fruits [10]. Although the highly homologous Japanese cedar allergen Cry $\mathrm{j} 1$ was found to have pectate lyase activity [11], Jun a 1 has not yet demonstrated more than minimal enzymatic activity, relative to the microbial pectate lyases [5]. The homologous bacterial Pnl and Pel proteins contain two highly conserved sequences, $\mathrm{vWiDH}$ and $\mathrm{RxPxxR}$, along with a characteristic $\beta$-helical core [12], all of which are also present in Jun a 1 . The low level of enzymatic activity of Jun a 1 has been hypothesized to be due to steric hindrance of the enzyme's active site or obliteration of the substrate binding groove [5]. Unique to Jun a 1 , the first 30 residues of the $\mathrm{N}$ terminus cover the conserved site vWiDH by making a complex loop. Using more sensitive assays, we herein provide evidence that Jun a 1 does indeed have pectate lyase activity, but at levels that are less than other plant homologues and far less than bacterial pectate lyases. We have therefore studied mutations of two potentially critical regions in rJun a 1 .

A heterologous expression system would also allow the elucidation of the structure-function relationships of the allergenicity of Jun a 1 and the development of rJun a 1 for diagnostic and therapeutic applications. Subcutaneous immunotherapy with crude extracts from cedar has met with limited success, with only $30 \%$ of the patients responding after 2 years of weekly injections [13]. Mutagenesis of rJun a 1 could be used to complete the immunological analysis of this important allergen by defining the location of discontinuous epitopes for IgE and IgG antibodies [14]. A more complete understanding of the immunogenicity of Jun a 1 could aid in the development of a Jun a 1-based vaccine for immunotherapy that causes less clinical reaction, thereby allowing more rapid dose escalation.

We report here the successful expression of run a 1 in the tobacco Nicotiana benthamiana using a TMV vector to achieve yields of $100 \mu \mathrm{g} / \mathrm{g}$ leaf material. Secretion to the apoplast (interstitial area) followed by collection by vacuum infiltration contributed to the purity of Jun a 1 in the crude extract. We also demonstrated the antigenicity and pectate lyase activity of the recombinant allergen and present preliminary findings from Jun a 1 mutants.

\section{Materials and Methods}

\section{Construction of Jun a 1 Mutants and Insertion into TMV \\ Vector}

All constructs were based on the TMV binary plasmid expression vector JL36 [15], which is delivered via agroinfection. pJL36 (fig. 1) contains the full complement of TMV genes as well as an additional subgenomic promoter with a multiple cloning site (PacI, AvrII and NotI). PCR with mutated primers was used to add PacI and AvrII sites to the termini of the wild-type and mutant Jun a 1 ORFs, including the native signal peptide, using as template Jun a 1 cDNA described by Midoro-Horiuti et al. [3]. Jun a 1 ORF mutants were created by the NIEHS Center Molecular Genetics Core at the University of Texas Medical Branch. These included the $\mathrm{His}^{203}$ to Ala point mutation and the truncation of the 38-base N-terminal amino acids, as well as combinations of the two. The mutant ORFs were then cloned into PacI-AvrII-digested JL36 to create Jun a 1/JL36 (fig. 1). Phusion DNA polymerase (NEB, Beverly, Mass., USA) was used according to company protocols. Recombinant E. coli clones were screened by PCR using Taq polymerase (NEB) or restriction digest and then sequenced using a CEQ capillary sequencer (Beckman Coulter, Fullerton, Calif., USA).

Agroinoculation of $\mathrm{N}$. benthamiana Plants

Agrobacterium tumefaciens LBA4404 was used for the agroinoculation of N. benthamiana. A. tumefaciens LBA4404 was transformed with JL36/Junal vectors by electroporation at $1.44 \mathrm{kV}$ and $129 \Omega$ for 5 ms using a BTX 600 Electro Cell Manipulator (BTX Inc., San Diego, Calif., USA). A. tumefaciens transformants were selected with appropriate antibiotics and colonies were grown to an $\mathrm{OD}_{600}$ of 1.0 in liquid Luria-Bertani (LB) medium supplemented with $10 \mathrm{mM}$ MES ( $\mathrm{pH}$ 5.7), antibiotics and $20 \mu \mathrm{M}$ acetosyringone. Cells were collected by centrifugation and resuspended in $10 \mathrm{mM}$ MES (pH 5.7), $10 \mathrm{mM} \mathrm{MgCl}$ and $200 \mu \mathrm{M}$ acetosyringone [15]. After 2-8 h room temperature incubation, $N$. benthamiana leaves were infiltrated at a scratched spot on the abaxial surface using a 3-ml syringe with no needle.

Detection of Viral RNA and Extraction of Jun a 1 Protein

Symptoms of infection were visible after 5-6 days as mild leaf deformation in systemically infected leaves. At 7-12 days postinoculation, as necrosis commenced, systemic leaves (approx. 500 


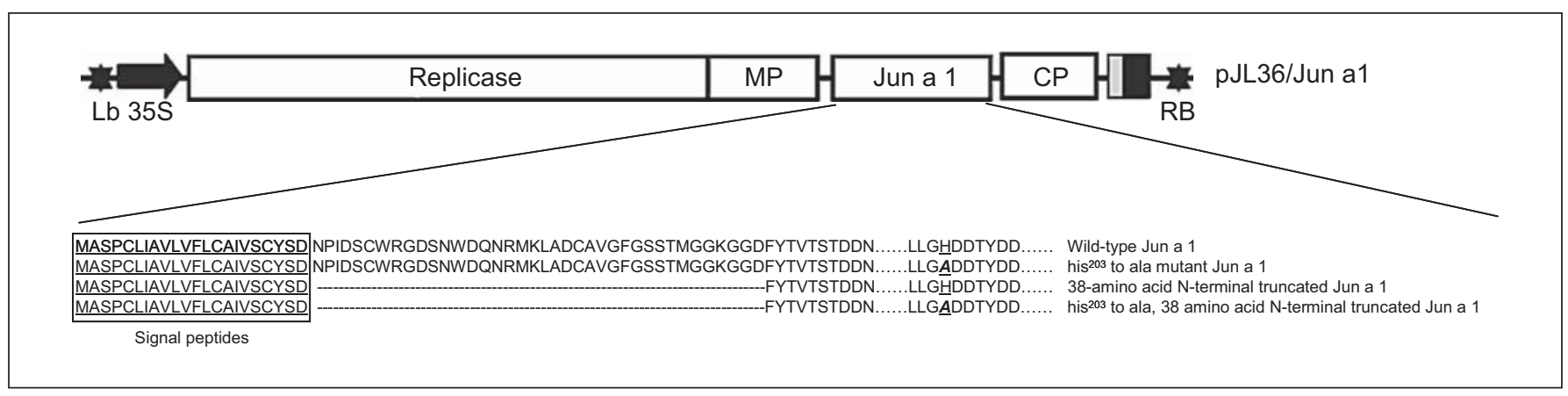

Fig. 1. Jun a 1 wild-type and mutant ORFs in the TMV vector pJL36 [15]. The left and right T-DNA borders (LB and RB) allow vector insertion into the plant chromosome following agroinfection. The CaMV 35S plant promoter allows production of a viral mRNA, which then replicates extensively in the cytoplasm. A viral subgenomic promoter drives Jun a 1 expression. The four Jun a 1 mutant insertions are shown below, including wild-type rJun a $1, \mathrm{His}^{203}$ to Ala mutant rJun a 1,38 amino acid N-terminal truncated rJun a 1 and 38 amino acid N-terminal truncation with $\mathrm{His}^{203}$ to Ala mutant rJun a 1 . Black arrow $=$ CaMV 35S promoter; gray box $=$ ribozyme; black box $=35 \mathrm{~S}$ terminator. TMV ORFs: replicase, movement protein (MP), coat protein (CP). mg) from each plant were harvested for total RNA extraction with TRI Reagent (Sigma, St. Louis, Mo., USA) followed by RT-PCR using screening primers (5'J1sigScr: 5'ATGGCTTCACCATGCTTAAT3' and 3'J1Scr: 5'TTAGGTTACAACTCCAGCAT3'). At 11-14 days postinoculation, systemic leaf and stem material was harvested, weighed, and then subjected to a 700-mm Hg (93$\mathrm{kPa}$ ) vacuum for $2 \mathrm{~min}$ in infiltration buffer [100 mM Tris ( $\mathrm{pH}$ 7.5), $10 \mathrm{mM} \mathrm{MgCl}_{2}, 2 \mathrm{~mm}$ EDTA] [16]. Secreted proteins were then recovered from infiltrated leaves by centrifugation at 2,000 $\mathrm{g}$ with the leaves supported on metal sink strainer cups which fit onto the mouths of Nalgene $250-\mathrm{ml}$ wide-mouth centrifuge bottles. The apoplastic fluid, containing recombinant protein, was collected at the bottom of the bottles while the leaves remained intact in the top strainer cup.

\section{Detection of Pectate Lyase Activity by Capillary Zone}

Electrophoresis

A mixture of galacturonic acid oligomers was produced by autoclave hydrolysis of pectic acid as described by Robertsen (K174001). GalA 9 was purified from the mixture by chromatography on a $22 \times 250 \mathrm{~mm}$ PA1 anion exchange column (Dionex Corp., Sunnyvale, Calif., USA) as described previously [17]. The GalA, oligomer was labeled with aminopyrene trisulfonic acid and purified away from labeling reagents following procedures of Naran et al. [18]. To test for lyase activity, $10 \mu \mathrm{l}$ of $0.1 \mathrm{M}$ Tris buffer ( $\mathrm{pH}$ 8.5), $1 \mu \mathrm{l}$ of $5 \mathrm{mM} \mathrm{CaCl}_{2}, 1 \mu \mathrm{l}$ apoplastic fluid, or native Jun a 1 , $8 \mu \mathrm{l}$ water, and $1 \mu \mathrm{l}$ fluorescent substrate were incubated in a 0.5 $\mathrm{ml}$ microfuge tube at room temperature. The concentration of fluorescent substrate was chosen so that a signal intensity of approximately 20 relative fluorescence units was observed for the undigested substrate under the capillary zone electrophoresis conditions we used. After various lengths of time the sample was analyzed on a BioFocus 2000 (Bio-Rad Laboratories, Hercules, Calif., USA) with laser-induced fluorescence detection. A fusedsilica capillary (TSP050375; Polymicro Technologies, Phoenix, Ariz., USA) of internal diameter $50 \mu \mathrm{m}$ and length $31 \mathrm{~cm}$ was used as the separation column. The samples were injected by application of 4.5 psi of helium pressure for $0.22 \mathrm{~s}$. Electrophoresis condi- tions were $15 \mathrm{kV} / 70-100 \mu \mathrm{A}$ with the cathode at the inlet, $0.1 \mathrm{M}$ sodium phosphate, $\mathrm{pH} 2.5$, as running buffer, and a controlled temperature of $20^{\circ} \mathrm{C}$. The capillary was rinsed with $1 \mathrm{~N} \mathrm{NaOH}$ followed by running buffer between injections. Oligomers labeled with aminonaphthalene trisulphonic acid (APTS) were excited at $488 \mathrm{~nm}$ and emission was collected through a $520-\mathrm{nm}$ band pass filter.

\section{Detection of Pectate Lyase Activity by Mass Spectrometry}

Five hundred micrograms of galacturonic acid oligomer, $\mathrm{GalA}_{9}$, was dissolved in $25 \mu \mathrm{l} 1 \times \mathrm{PBS}$ buffer ( $\mathrm{pH}$ 7.4) and mixed with $0.5 \mu \mathrm{l} 5 \mathrm{mM} \mathrm{CaCl}_{2}$ and $2.5 \mu \mathrm{g} / 25 \mu \mathrm{l}$ of rJun a 1 . Two microliters of toluene were added to prevent microbial growth. The progress of the digestion was followed by taking 1- $\mu$ l aliquots, labeling the oligomers with APTS and analysis by capillary zone electrophoresis. After 4 days incubation at room temperature the oligomers were desalted by passing through a Shodex protein KW-802.5 gel permeation column (Shodex Inc., New York, N.Y., USA) in $200 \mathrm{mM}$ ammonium acetate buffer with detection via a refractive index monitor. Oligosaccharide fractions were analyzed using a Voyager De-Pro MALDI-TOF mass spectrometer (Applied Biosystems, Foster City, Calif., USA) in the negative ion reflector mode with a matrix of $100 \mathrm{mg} / \mathrm{ml}$ trihydroxyacetophenone in methanol.

\section{Purification of rJun a 1}

For some of the analyses, rJun a 1 was purified by affinity column chromatography on a 1-ml HiTrap NHS-HP column (Sigma) labeled with a cocktail of anti-Jun a 1 monoclonal antibodies (L19a-4, L2b-31, L27a-8, 56-h-58 and 70-7-18L-9). Apoplastic rJun a 1 was first centrifuged and then filtered $(0.45 \mu \mathrm{m})$. The column was washed and equilibrated with binding buffer [PBS ( $\mathrm{pH} 7.4)$, $0.01 \% \mathrm{NaN}_{3}$ ] prior to injecting the apoplastic lysate with a final flow rate of $0.2 \mathrm{ml} / \mathrm{min}$. Elution of the bound rJun a 1 was performed with $0.2 \mathrm{M}$ glycine- $\mathrm{HCl}(\mathrm{pH} 2.6)$ and captured in 1-ml fractions. Each of these fractions were then analyzed by SDS polyacrylamide gel (4-20\%; Bio-Rad) electrophoresis and subsequently stained with Coomassie brilliant blue R-250 (Sigma). The con- 
centrations of purified proteins were estimated by densitometry of the Comassie-stained gel and compared with dilutions of bovine serum albumin (BSA) on the same gel (NEB).

\section{SDS-PAGE and Immunoblotting of rJun a 1}

Total plant extracts of Jun a 1 and purified rJun a 1 were separated on $4-20 \%$ Tris-HCl PAGE gels (Bio-Rad) and subsequently stained with Coomassie blue R-250. For immunoblotting experiments, rJun a 1 was electroblotted from SDS-PAGE gels onto a PVDF membrane (Amersham, Piscataway, N.J., USA) by semidry transfer cells (Bio-Rad). The blot was blocked with TBST buffer ( $100 \mathrm{~mm}$ Tris- $\mathrm{HCl}, 0.9 \% \mathrm{NaCl}, 0.1 \%$ Tween 20 , pH 7.5) containing $10 \% \mathrm{BSA}$ at room temperature for $1 \mathrm{~h}$. The membranes were then incubated, at $4{ }^{\circ} \mathrm{C}$ over night, with a mixture of $0.5 \mu \mathrm{g} / \mathrm{ml}$ each of anti-Jun a 1 monoclonal antibodies (L1b-18, L86-10, L27b21, L20-15, and KW-S91 anti-Cry j 1 antibody; KW-S91 was a gift of M. Watanabe, PhD, New Drug Research Laboratories II, Kowa Co. Ltd., Tokyo, Japan). Bound mouse IgG was detected by biotinylated goat anti-mouse IgG and horseradish peroxidase (HRP)streptavidin (Zymed, San Francisco, Calif., USA) [3]. Visualization was performed via ECL detection reagents (Amersham) followed by photometric detection.

\section{IgE Binding to Native (nJun a 1) and rJun a 1 Allergens}

IgE binding to Jun a 1 was measured by ELISA. Three serum samples were obtained from $J$. ashei-allergic patients from the area of Austin, Texas, USA. ELISA plates were coated with $3 \mu \mathrm{g} /$ $\mathrm{ml}$ nJun a 1 or rJun a 1 in borate buffer $(0.125 \mathrm{M}$ boric acid, $\mathrm{pH} 8.8$, $0.225 \mathrm{M} \mathrm{NaCl}, 5 \mathrm{~mm}$ EDTA) at $37^{\circ} \mathrm{C}$ over night. Patients' sera were serially diluted $(1: 4 ; 1: 16$ and $1: 64)$ with PBS containing $0.05 \%$ Tween 20 (TPBS) on the plates and incubated at room temperature for $2 \mathrm{~h}$. Detection of bound Jun a 1-specific IgE was achieved by incubating the plates with biotin-conjugated goat anti-human IgE (1:300 in TPBS; Sigma) and HRP-streptavidin (1:1,000 in TPBS; Sigma) with shaking at room temperature for $60 \mathrm{~min}$. Colorimetric substrate, FAST OPD (Sigma), was added and incubated for $20 \mathrm{~min}$ at room temperature. The reaction was stopped and $\mathrm{OD}_{492}$ was quantified in each well on a plate spectrophotometer (SoftmaxPro 4.0 plate spectrophometer; Molecular Devices, Sunnyvale, Calif., USA).

\section{Monoclonal Anti-nJun a 1 Antibody Binding to nJun a 1 and} rJun a 1

ELISA plates were coated with $3 \mu \mathrm{g} / \mathrm{ml}$ nJun a 1 or rJun a 1 as described above. Mouse monoclonal IgG antibodies serially diluted to $1,000,100$ and $10 \mathrm{ng} / \mathrm{ml}$ in TTBS were added to duplicate wells on both plates and incubated at room temperature for $2 \mathrm{~h}$. Bound IgG was detected by HRP-conjugated goat anti-mouse IgG $(1: 1,000$ in TPBS) at room temperature for $1 \mathrm{~h}$. The bound enzyme activity was quantified as described above.

\section{Comparison of the Display of Human IgE Epitopes on rJun a 1} and nJun a 1

Epitope display was assessed by inhibition assays. Ninety-sixwell ELISA plates were coated with purified native Jun a 1 or leaf lysate containing $3 \mu \mathrm{g} / \mathrm{ml} \mathrm{rJun}$ a 1 , as described above. Equal volumes of solutions containing varying concentrations of rJun a 1 or nJun a 1 ( 0 to $50 \mu \mathrm{g} / \mathrm{ml})$ or a negative control solution containing lysates from leaves infected with the same vector containing an insert encoding GFP were added to a mixture of sera from 3 cedar pollinosis patients (equal volume of each). The mixtures were incubated at room temperature for $30 \mathrm{~min}$ and added to the coated plates. These plates were incubated at room temperature for $1 \mathrm{~h}$ with shaking. The ability of the two forms of Jun a 1 to inhibit the binding of human $\operatorname{IgE}$ was assessed by detecting the amount of human IgE bound to the plates using the detection system described above. The percent inhibition was computed by comparison with wells that contained a mixture of the patient's sera and buffer rather than Jun a 1 solutions.

\section{RBL SX-38 Cell Culture and IgE-Dependent}

$\beta$-Hexosaminidase Release Assay

Rat basophilic cell line, RBL SX-38, which expresses the $\alpha$ - and $\beta$-chains of the human FceRI (a gift from J.-P. Kinet, Harvard University) [19], were cultured in DMEM with $10 \%$ fetal calf serum, $1 \%$ penicillin/streptomycin and $1.2 \mathrm{mg} / \mathrm{ml} \mathrm{G} 418$ (Invitrogen, Carlsbad, Calif., USA) [20]. Cells were subcultured in 96-well plates at $1 \times 10^{5}$ cells/well in DMEM without G418, at $37^{\circ} \mathrm{C}$, in $5 \%$ $\mathrm{CO}_{2}$ over night and the wells were washed with Hanks solution without $\mathrm{Mg}^{++}$and $\mathrm{Ca}^{++}$. The cells bound to the wells were sensitized with serum (1:10 dilution) from individual mountain cedarsensitive patients, $100 \mu \mathrm{l} /$ well, at $37^{\circ} \mathrm{C}$, in $5 \% \mathrm{CO}_{2}$ over night. Cells were washed with $100 \mu \mathrm{l} /$ well of warmed HEPES-Tyrode's buffer (137 mM NaCl, 5.6 mM glucose, $2.7 \mathrm{~mm} \mathrm{KCl,} 0.5 \mathrm{~mm} \mathrm{NaH}$ ${ }_{2} \mathrm{PO}_{4}, 1 \mathrm{mM} \mathrm{CaCl}_{2}, 10 \mathrm{~mm}$ HEPES and 0.1\% BSA, pH 7.3) 3 times. Duplicate wells were challenged with $0.5-50 \mathrm{ng} / \mathrm{ml}$ purified recombinant or native Jun a 1 or A23187 in Tyrode's buffer as a negative control and incubated at $37^{\circ} \mathrm{C}$, in $5 \% \mathrm{CO}_{2}$, for $30 \mathrm{~min}$. Twenty-five microliters of the supernatants from each well were transferred to a new 96-well plate. Five microliters of $1.2 \%$ Triton $\mathrm{X} 100$ and $30 \mu \mathrm{l}$-nitrophenyl-N-acetyl- $\beta$-D-glucopyranoside (NAG; Sigma) were added to the assay wells at the same time. The NAG was made with 4 volumes of $0.01 \mathrm{M}$ NAG and 1 volume of $0.4 \mathrm{M}$ citrate buffer ( $\mathrm{pH} 4.5$ ). The 96 -well plates were shaken at $37^{\circ} \mathrm{C}$ for $2 \mathrm{~h}$. To stop the $\beta$-hexosaminidase reaction, $20 \mu \mathrm{l} 1 \mathrm{~N}$ $\mathrm{NaOH}$ were added to each well. The plate was read at $410 \mathrm{~nm}[21$, 22] using a SoftmaxPro 4.0 plate spectrophometer (Molecular Devices). Results are reported as percentage of the total $\beta$-hexosaminidase released after the addition of $1 \%$ Triton X-100.

\section{Results}

\section{Construction of TMV/Jun a 1 Vector and Expression in N. benthamiana Plants}

The wild-type and mutant Jun a 1 coding sequences, including its signal peptide, were successfully amplified and inserted into the pJL36 vector under the control of the TMV-U1 coat protein subgenomic promoter (fig 1). The variants were designed to test whether or not a salt bridge (His ${ }^{203}$ to Ala mutant), which was predicted to obstruct the substrate grove of Jun a 1, or an N-terminal extension (N-term truncation mutant), which was predicted to mask the pectolytic site $\left(\mathrm{Asp}^{177}, \mathrm{His}^{203}\right.$ and $\left.\mathrm{Arg}^{229}\right)$, inhibited pectate lyase activity [5]. Insertion of the genes, as well as correct orientation, was verified by 


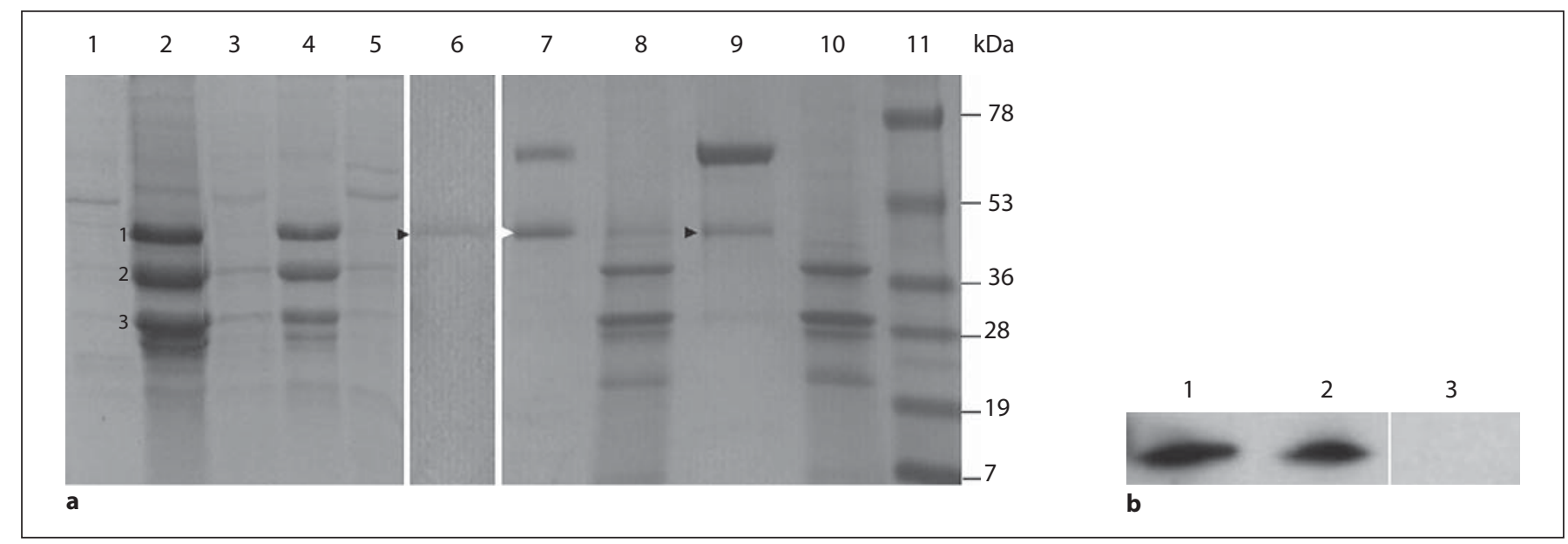

Fig. 2. Analysis of rJun a 1 protein expression and purification. a SDS-PAGE/Coomassie Blue-stained gel of apoplastic extracts of $N$. benthamiana infected with viral vectors. (1) Uninoculated negative control, (2) wild-type rJun a 1, (3) N-terminal truncated rJun a $1,(4) \mathrm{His}^{203}$ to Ala mutant rJun a $1,(5) \mathrm{His}^{203}$ to Ala/N-terminal truncated rJun a 1, (6) purified recombinant Jun a 1, (7) purified nJun a 1 with protective BSA, (8) unpurified rJun a 1, (9) purified rJun a 1 with protective BSA, (10) flow-through of affinity purifi-

PCR and DNA sequencing. Seven days after inoculation, total RNA was isolated from the systemically infected leaves of the host plant, $N$. benthamiana. RT-PCR products confirmed that the Jun a 1 sequence was stably maintained in the TMV vector in the systemically-infected leaves of the host plant, $N$. benthamiana.

Both wild-type and the $\mathrm{His}^{203}$ to Ala mutant of Jun a 1 protein were expressed in good quantity in $N$. benthamiana plants. These proteins were secreted into the apoplast (interstitial area) and were collected by vacuum infiltration of detached leaves with buffer, followed by a low-speed centrifugation to collect the apoplastic fluid (essentially, dew). SDS-PAGE revealed a major Coomassie-Blue-stained band migrating at $44 \mathrm{kDa}$ for the wildtype Jun a 1 plants, which was not present in the empty vector or uninfected control plants (fig. 2a). This $44-\mathrm{kDa}$ protein had identical migration in SDS-PAGE as nJun a 1 (fig. 2a). We confirmed that the recombinant protein was Jun a 1 by MALDI-TOF analysis and by N-terminal sequencing, which also revealed that the native signal peptide was correctly eliminated by the plant. The $r$ Jun a 1 was expressed at approximately $100 \mu \mathrm{g}$ from the vacuum infiltrate of $1 \mathrm{~g}$ of intact leaves, as estimated by densitometry of SDS-PAGE containing serially diluted BSA standards. When rJun a 1 was purified from apoplastic extracts by HiTrap NHS-HP monoclonal affinity column cation column, (11) protein molecular weight standard in kDa. White arrow $=$ nJun a 1 ; black arrow $=$ purified rJun a 1 . MALDITOF analysis (Lane 2): band 1, Jun a 1; band 2, $\beta$-1,3-glucanase; band 3, chitinase. b Western blot: (1) wild-type Jun a 1, (2) $\mathrm{His}^{203}$ to Ala mutant Jun a 1, (3) uninoculated negative control. Western blot was probed with a mixture of anti-Jun a 1 monoclonal antibodies: L1b-18, L86-10, L27b-21, L20-15, and KW-S91 anti-Cry j 1 antibody. chromatography (fig. 2b), a yield of $6 \mu \mathrm{g}$ rJun a 1/g of leaf tissue was achieved. The rJun a 1 protein was totally removed from the flow-through from the purification column (fig. 2a). These results were confirmed by Western blotting with anti-Jun a 1 antibodies (fig. 2b).

\section{Plant Necrosis and Jun a 1 Mutant Analysis}

Typical symptoms of viral infection were seen in all infected plants, with mild leaf deformation at 5-6 days postinoculation and systemic mosaic symptoms by 10 days postinoculation. However, in wild-type Jun a 1-infected plants, necrotic patches began to appear at 12 days postinoculation in the inoculated and systemic leaves, followed by necrosis of the entire plant at 20-30 days postinoculation (fig. 3). Protein was collected 11-14 days after inoculation in systemic leaves before the most severe necrosis occurred. Associated with this necrosis was the presence of two extra protein bands in addition to the Jun a 1 band in SDS-PAGE analysis (fig. 2). These were identified by MALDI-TOF analysis as the common apoplastic pathogenesis-related proteins, $\beta$-1,3-glucanase and chitinase. These proteins were probably produced in response to the necrosis induced by the Jun a 1 expressed from the TMV vector.

Necrosis was seen in all plants expressing Jun a 1 protein (fig. 3a, c) and was never seen in plants that did not 

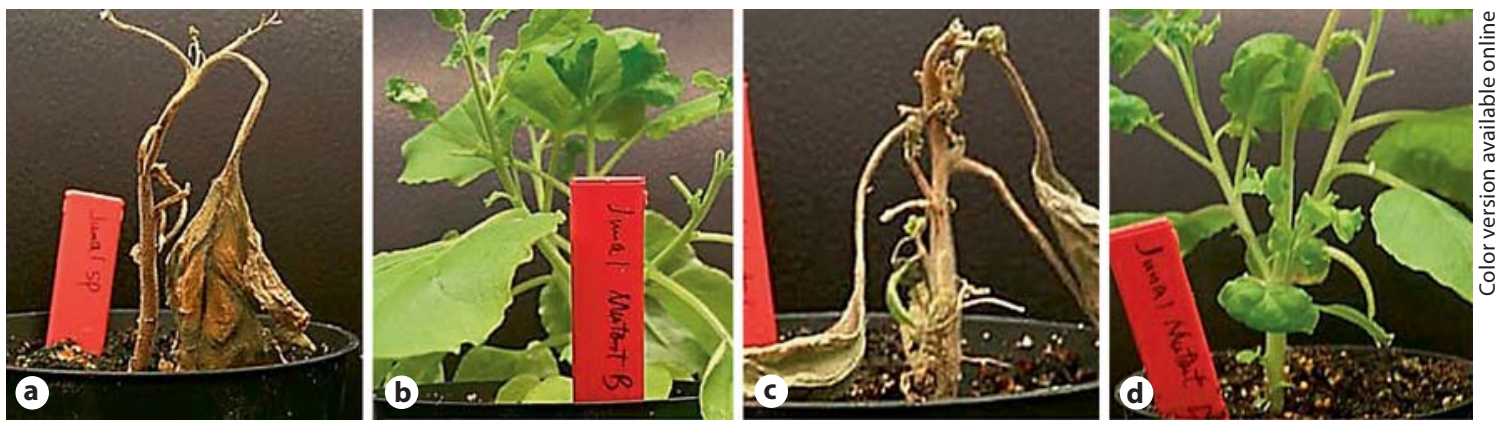

Fig. 3. Symptoms of plants expressing rJun a 1 from the TMV viral vector ( 30 days postinoculation). a Wildtype Jun a 1; b 38 amino acid N-terminal truncated Jun a 1; $\mathbf{c ~ H i s}{ }^{203}$ to Ala mutant, full-length Jun a 1; d His ${ }^{203}$ to Ala, N-terminal truncated Jun a 1. All plants were shown by RT-PCR to be infected with the viral vector (data not shown).

express Jun a 1 (empty vector controls and mutants shown in fig. 3b, d). An His ${ }^{203}$ to Ala mutant was created in order to remove the histidine-aspartate bridge which is thought to collapse the pectate lyase substrate groove of Jun a 1 [5]. Symptoms developing on these plants were largely indistinguishable from those on wild-type-inoculated plants (fig. 3a, c) and similar quantities of 44-kDa protein were detected by SDS-PAGE. However, none of the plants inoculated with either of the mutant viruses harboring the truncation mutation (fig. 3b, d) showed symptoms or expressed Jun a 1 protein. As a positive control for a role of pectate lyase in the plant necrosis, a pectate lyase gene from banana fruit (a kind gift from Dr. Marín-Rodríguez) was inserted into the TMV vector. The necrotic symptoms induced with this construct perfectly paralleled those developed by TMV/Jun a 1-inoculated plants in time course and severity (data not shown). Recombinant banana pectate lyase enzyme has previously been shown to possess pectate lyase activity [10].

\section{Detection of Pectate Lyase Activity by Capillary Zone Electrophoresis}

The favorable comparison in symptoms developed by rJun a 1 and banana pectate lyase led us to suspect that Jun a 1 might also possess pectate lyase activity, which would degrade the adhesive pectin layer between the plant cells, resulting in the necrotic (or perhaps apoptotic) response seen.

One microliter containing $1 \mu \mathrm{g}$ of native Jun a 1 protein purified from pollen was incubated with a small amount of a fluorescently labeled nonamer of galacturonic acid $\left(\mathrm{GalA}_{9}\right)$. The oligomer incubated with Jun a 1 was converted into shorter oligomers in a time-dependent manner. After only $30 \mathrm{~min}$, about half of the oligo- mer had been converted to smaller products (fig. 4). The same products were produced at a slower rate by our rJun a 1 from N. benthamiana and by the $\mathrm{His}^{203}$ to Ala mutant (fig. 5). Pectate lyase activity in fluids from leaves inoculated with the empty vector control was not detected after $1 \mathrm{~h}$ of incubation (fig. 5). No pectate lyase activity was detected in the uninoculated $N$. benthamiana apoplast control sample, although a low amount of exopolygalacturonase activity was observed. This activity became much more pronounced when the incubation buffer was $50 \mathrm{~mm}$ ammonium acetate ( $\mathrm{pH}$ 4.5). Significant exopolygalacturonase activity in apoplastic fluids from cotton cotyledons has been observed previously [23].

To verify that the enzyme activity was actually due to a pectate lyase, a larger sample of unlabeled $\mathrm{GalA}_{9}$ was digested with the wild-type Jun a 1 from $N$. benthamiana and, after desalting, analyzed by MALDI-TOF mass spectrometry. As would be expected for the action of a pectate lyase, a mixture of saturated and unsaturated oligomers was produced. For example, after cleavage by a lyase of the $\mathrm{GalA}_{9}$ oligomer between the fourth and fifth sugars in the chain, a $\mathrm{GalA}_{4}$ oligomer and a $\mathrm{GalA}_{5}$ oligomer with a 4,5 unsaturated GalA at its nonreducing end would be produced. If the enzyme cleaved between the fifth and sixth residues, a GalA $\mathrm{A}_{5}$ oligomer and an unsaturated $\mathrm{GalA}_{4}$ oligomer would result. Ions were observed corresponding to saturated GalA oligomers containing 3 to 9 residues minus a proton $(\mathrm{m} / \mathrm{z}=545.17,721.25,897.29$, $1,073.36,1,249.38,1,425.44$, and 1,601.51), and to unsaturated oligomers of $4-8$ GalA residues $(\mathrm{m} / \mathrm{z}=703.22$, $879.29,1,055.33,1,231.38$ and 1,407.38) (fig. 6). From this combination of products one can infer that the enzyme has endo-pectate lyase activity. 


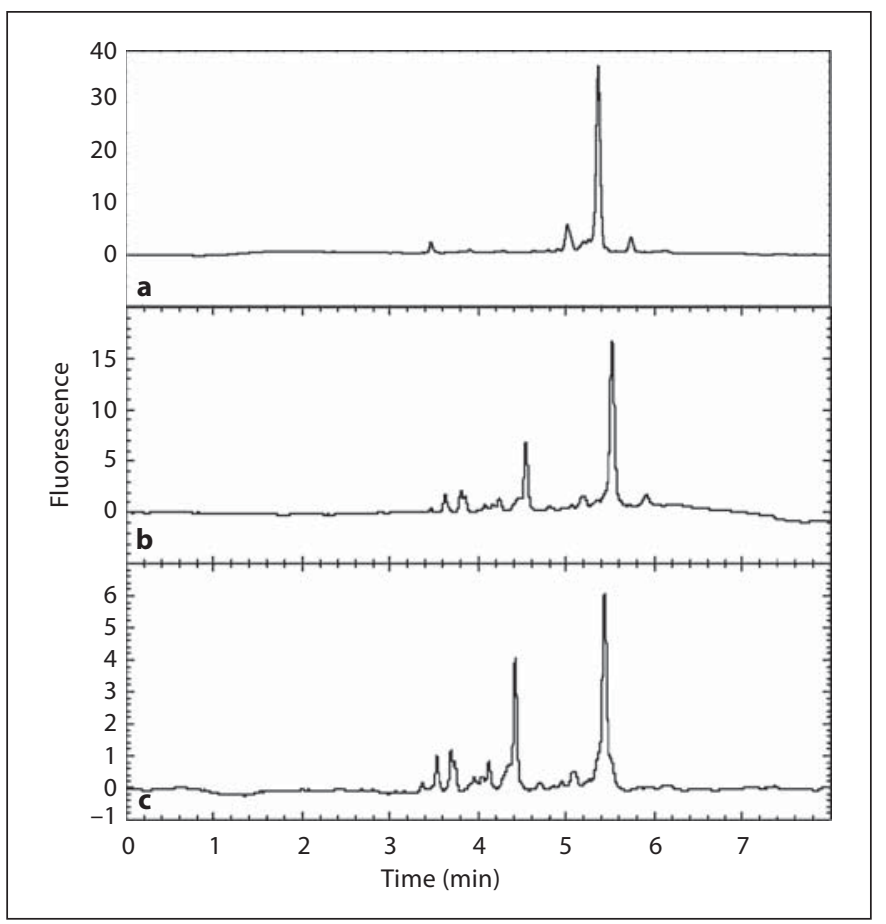

Fig. 4. Electropherograms of the reaction products between fluorescently labeled GalA, substrate and nJun a 1. a Substrate alone; b 30 min incubation with Jun a 1 protein; c 60 min incubation.

From the length of time it took the $2.5 \mu \mathrm{g}$ of Jun a 1 to degrade $90 \%$ of the $500 \mu \mathrm{g}$ of GalA oligomer, we estimate that the enzyme has a specific activity of at least $0.2 \mathrm{mU} /$ $\mathrm{mg}$. This is comparable to the value for recombinant banana pectate lyase of $10.5 \mathrm{mU} / \mathrm{mg}$ protein (1 U forms 1 $\mu \mathrm{M}$ of uronide products $/ \mathrm{min}$ ) [10], but is nearly 2 millionfold lower than the $350 \mathrm{U} / \mathrm{mg}$ protein reported for Japanese cedar pollen pectate lyase, Cry j 1 [11].

\section{IgE-Binding Capacity of rJun a 1 Allergens in ELISA}

Assays

To test the extent to which the rJun a 1 produced in $N$. benthamiana retained the capacity of nJun a 1 to bind $\operatorname{IgE}$ from the sera of mountain cedar-allergic patients, serial dilutions of patient sera were tested in ELISA experiments in which the plates were coated with equivalent amounts of rJun a 1, nJun a 1 or lysates of uninfected leaves. For each antibody concentration, three replicates were performed and the data are presented as the mean values (fig. 7). The results demonstrate that IgE antibodies from the sera of three subjects with J. ashei pollinosis bound significantly better to lysates from leaves expressing rJun a 1 than to lysates prepared from leaves from

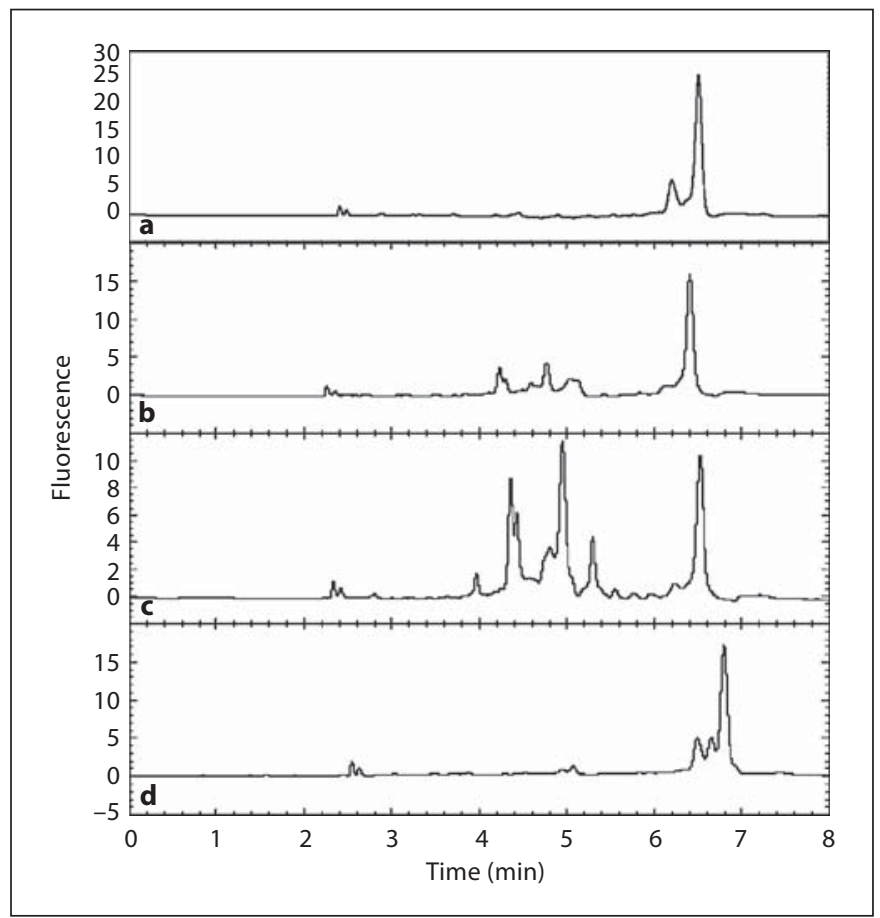

Fig. 5. Electropherograms of the reaction products after $1 \mathrm{~h}$ incubation of fluorescently labeled GalA, substrate and rJun a 1 from $N$. benthamiana. a Substrate alone; b the $\mathrm{His}^{203}$ to Ala mutant; c wild-type Jun a 1; d empty vector control.

uninoculated $N$. benthamiana plants. In addition, the relative amount of IgE binding to recombinant and nJun 1 varied from fully equivalent to approximately one fourth of the amount bound to nJun a 1 (fig. 7).

\section{Monoclonal Antibody Binding to nJun a 1 and rJun a 1 Allergens}

Monoclonal antibodies 7g-44, 39A-80, L-8b-10 produced in our lab against native Jun a 1 represent three antibody groups. Monoclonal antibodies $7 \mathrm{~g}-44$ and 39A80 recognize heat labile (conformational) epitopes, and L- $8 \mathrm{~b}-10$ recognizes a heat stable (linear) epitope. The data displayed in figure 8 shows that the Jun a 1 epitopes recognized by monoclonal antibodies 39A-80 and L-8b-10 are displayed equally well on recombinant and nJun a 1 , while the epitope defined by monoclonal antibody $7 \mathrm{~g}-44$ binding are not well displayed on rJun a 1 .

\section{Comparison of the Display of Human IgE Epitopes on}

rJun 1 and nJun a 1 by Inhibition Assays

Inhibition of IgE-binding was examined to determine the relative ability of native and recombinant Jun a 1 to bind an array of human anti-Jun a 1 antibodies in a pool 
Fig. 6. MALDI-TOF mass spectrum of the products of $\mathrm{GalA}_{9}$ digested with rJun a 1 from $N$. benthamiana. $\mathrm{m} / \mathrm{z}=545.17, \mathrm{GalA}_{3}$; 721.25, GalA ; 897.29, GalA $;$; 1,073.36, $\mathrm{GalA}_{6} ; 1,249.38, \mathrm{GalA}_{7} ; 1,425.44, \mathrm{GalA}_{8}$; $1,601.51, \mathrm{GalA}_{9}$. All ions resulting from loss of a proton. $\mathrm{m} / \mathrm{z}=703.22, \Delta \mathrm{GalA}_{4}$; 879.29, $\Delta \mathrm{GalA}_{5} ; 1,055.33, \Delta \mathrm{GalA}_{6}$; 1,231.38, $\Delta$ GalA $_{7} ; 1,407.38, \Delta$ GalA $_{8}$, where $\Delta$ indicates the 4,5 unsaturation of the GalA residue at the nonreducing end of the oligomer. Sodium adducts of many of the oligomers were seen at 22 mass units higher than the main peaks.

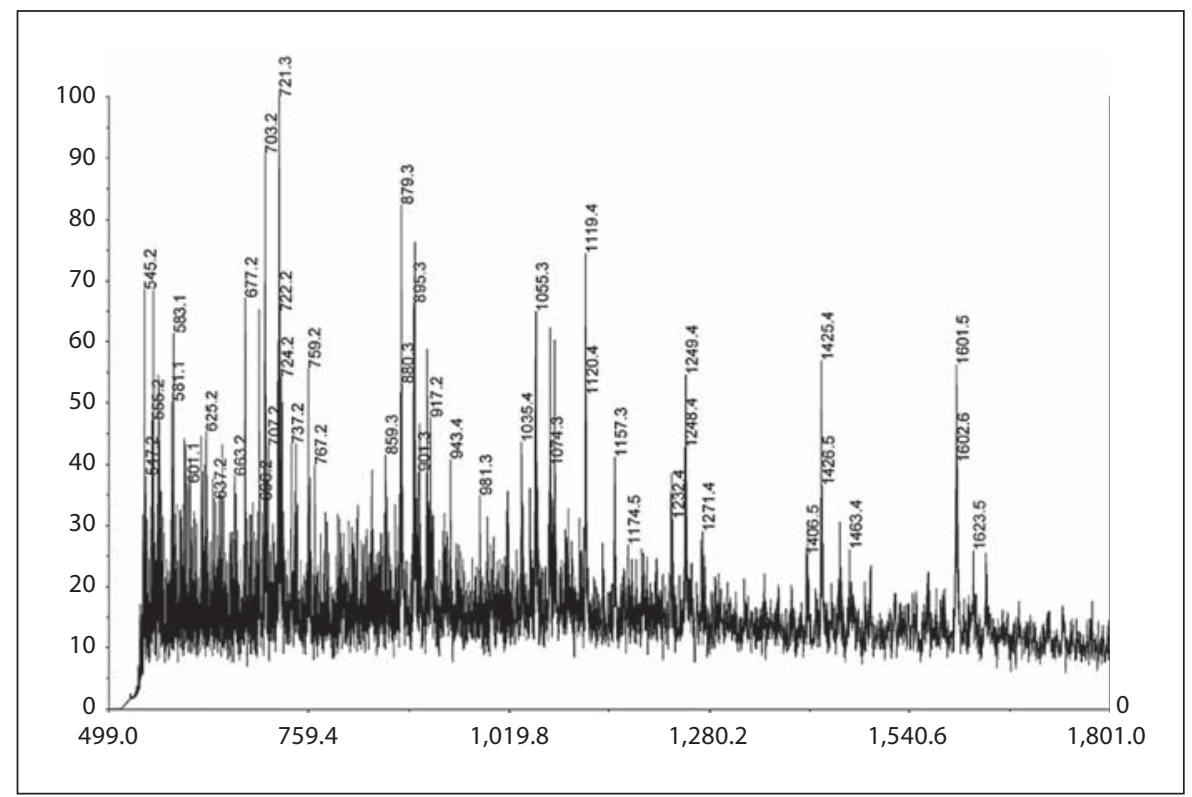

of sera from the same three patients of figure 7. The mixtures of serum and varying amounts of rJun a 1 or nJun a 1 (inhibitors) were tested on plates coated with rJun 1 and native Jun a 1 . As anticipated, the native and recombinant Jun a 1 produced a similar degree of inhibition when tested on the rJun a 1 plates (fig. 9). However, when the mixtures were tested on plates coated with nJun a 1 , the recombinant product only inhibited about half as much IgE binding as nJun a 1 did.

\section{Purified rJun a 1 Induces Degranulation of IgE-Sensitized RBL SX-38 Cells}

A mast cell-like RBL SX-38 cell line was used to predict the capacity of rJun a 1 to mediate an allergic reaction. The RBL SX-38 cells were sensitized with IgE antibodies from the same three sera from cedar pollinosis patients used in the ELISA assays shown in figure 7. The flowthrough buffer from the affinity purification column (fig. 2a) was used as a rJun a 1-negative control. Figure 10 shows that the purified recombinant allergens induced specific and dose-dependent $\beta$-hexosaminidase release from the sensitized mast cell-like cells.

\section{Discussion}

Jun a 1 is the dominant allergen of mountain cedar pollen, a major cause of allergy in the central USA. Jun a 1 has a high degree of sequence homology and cross-re- activity with the group 1 allergens from other cedar/cypress/juniper pollens. We have demonstrated here that Jun a 1 can be expressed relatively efficiently and in a soluble form in a TMV-based expression system. Further, we found that rJun a 1 in the leaf lysates were only contaminated with a few, largely plant pathogenesis related, proteins. For some of our studies we further purified the rJun a 1 by affinity chromatography, using a cocktail of monoclonal antibodies prepared by immunizing mice with nJun a 1 .

The immunologic reactivity of rJun a 1 with IgE antibodies from patients' sera was tested by three different types of assays: ELISA binding, allergen inhibition of human IgE binding and mast cell degranulation. While all three provided valuable information, the allergen-induced inhibition of IgE binding provided the most critical comparison of rJun a 1 and nJun a 1 reactivity. The results from the IgE ELISAs on plates coated with native and rJun 1 and the mast cell degranulation experiments indicated that rJun a 1 reacted with IgE from sera of all three patients, though they also suggested that rJun a 1 reactivity was less than that of nJun a 1 for the IgE in two of the sera. A comparison of the results from these two assays (fig. 7,10 ) for the three subjects suggests a relationship between the amount of IgE binding to Jun a 1 and the extent of mast cell degranulation, though the number of subjects is too small to fully test this proposition. The inhibition experiments were performed with a pool of sera from the same three subjects. The results from these 


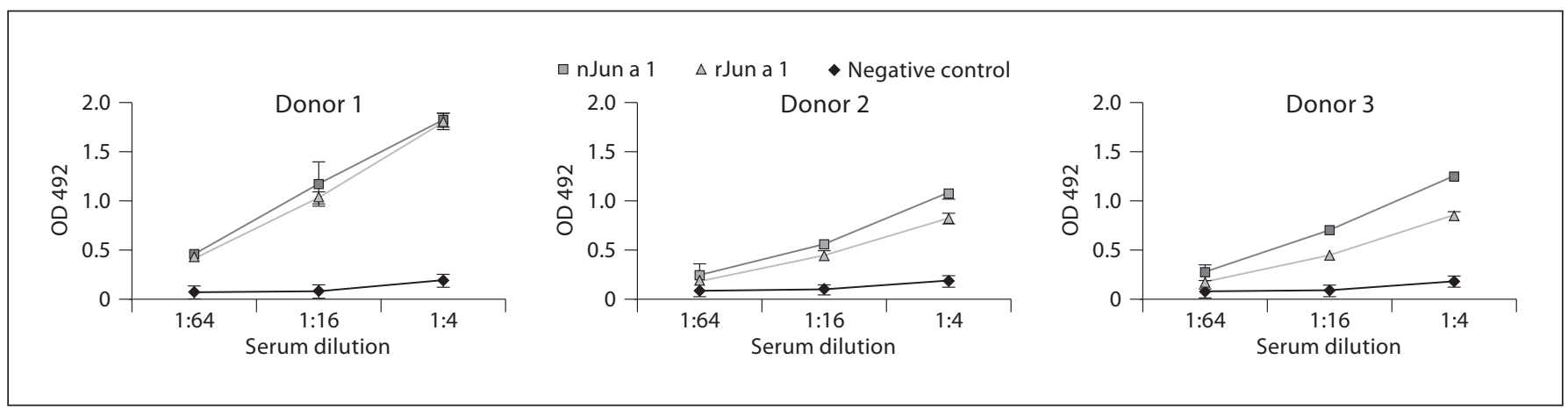

Fig. 7. Human IgE ELISA assay of nJun a 1 and rJun a 1 . IgE from the sera of three patients $(1,2$ and 3$)$ with mountain cedar allergy binding with native and recombinant wild-type Jun a 1 expressed in N. benthamiana, and with uninoculated $N$. benthamiana leaf lysate as negative control.

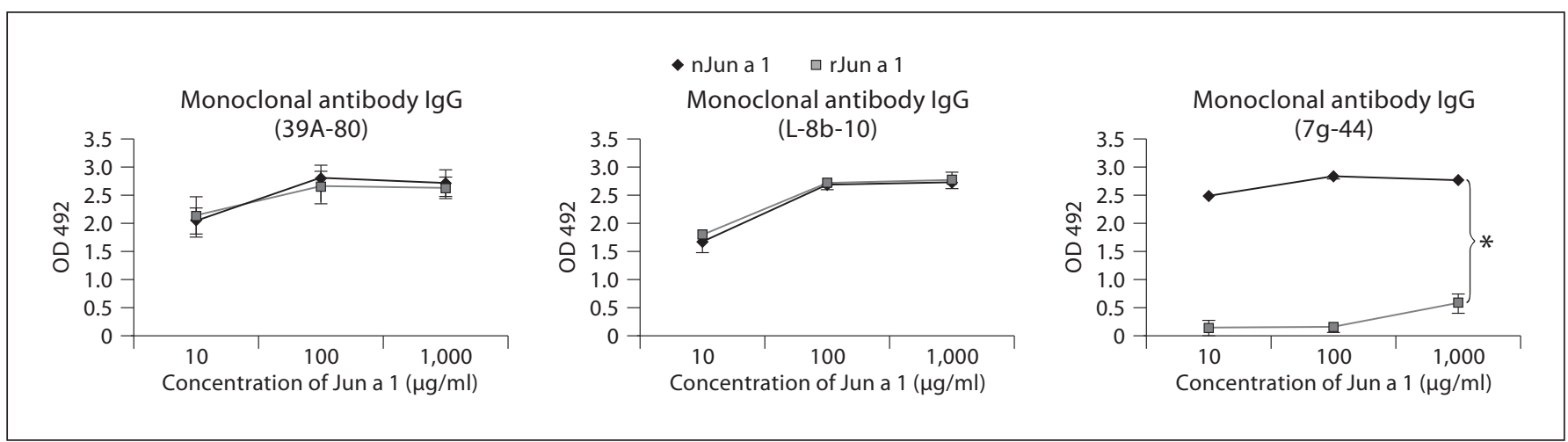

Fig. 8. Binding of monoclonal antibodies to nJun a 1 and rJun a 1 . Monoclonal antibodies 7g-44, 39A-80, L-8b10 binding with native and recombinant wild-type Jun a 1 expressed in $N$. benthamiana. Asterisk indicates a significant difference between binding to rJun a 1 and nJun a 1 ( $\mathrm{p}<0.05$, ANOVA).

assays performed on plates coated with nJun a 1 indicated that the rJun a 1 produced about half as much inhibition as the same concentrations of nJun a 1 . This could suggest that only one half of the major human IgE epitopes are displayed on the rJun a 1 or that all of epitopes were displayed, but only on about half as many rJun a 1 molecules. Preliminary analysis with our panel of monoclonal antibodies to nJun a 1 (fig. 8) suggests that some of the human IgE epitopes are displayed less well on rJun 1, compared to nJun a 1 , while others are equally well displayed on both forms. However, based on the results of the mast cell degranulation assay, the epitopes expressed on rJun a 1 are effective in cellular activation via IgE and the highaffinity IgE receptor. Thus, structural analysis of the epitopes that are differentially displayed on recombinant and nJun a 1 should help to elucidate the structure/function relationship of IgE epitopes of Jun a 1. Further, our current results indicate that the TMV expression system should provide meaningful results of site-directed and deletion mutational analyses of some important epitopes. The finding that nJun a 1 functioned as well as rJun a 1 in inhibition assays performed on rJun a 1-coated plates provides evidence that the IgE antibodies in our cedar pollinosis patient's sera do not bind to any of the tobaccoencoded proteins in the lysates from TMV-infected leaves. The same results also indicate that rJun al from leaf lysates do not excessively display human IgE epitopes.

The three-dimensional structure of Jun a 1 consists primarily of a series of parallel $\beta$-helices that is nearly identical to those found in the pectin/pectate lyases from several plant pathogenic microorganisms [5]. The sequences corresponding to the canonical $\mathrm{vWiDH}$ and $\mathrm{RxPxxR}$ sites are present in both pectin/pectate lyases 

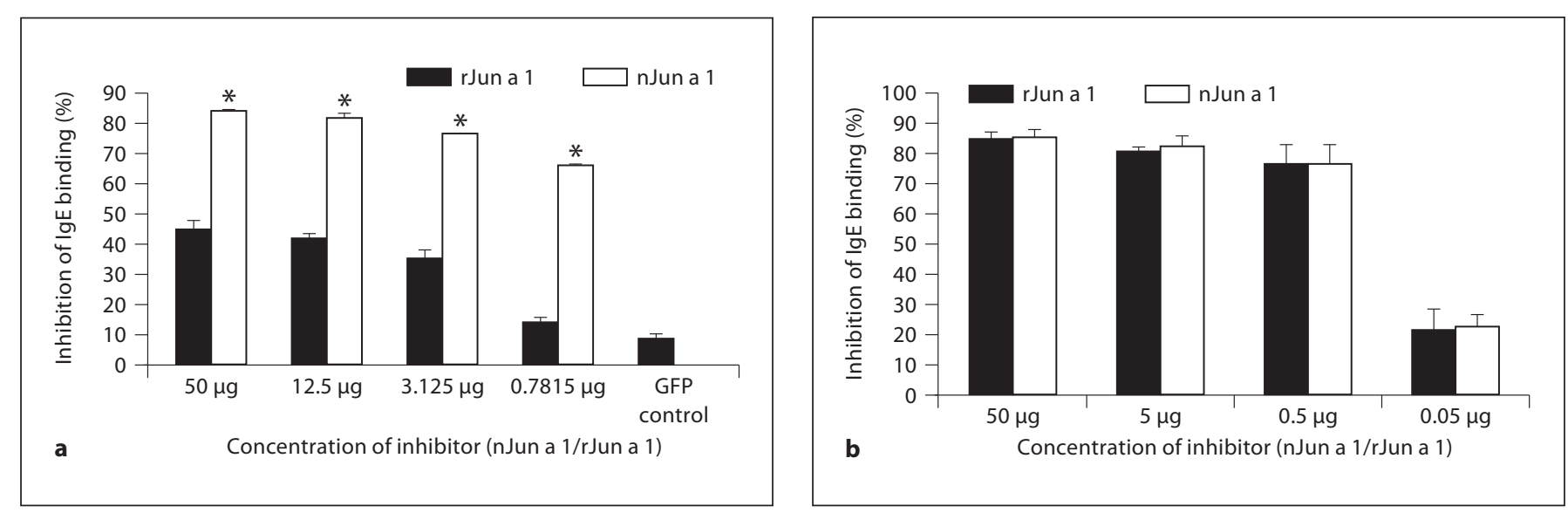

Fig. 9. Inhibition of human IgE binding to nJun a 1 (a) and rJun a 1 (b) in ELISA. The percentage inhibition was calculated using the OD readings from wells without inhibitor as $100 \%$. Asterisk indicates a significant difference between binding to rJun a 1 and nJun a 1 ( $\mathrm{p}<0.01$, ANOVA).

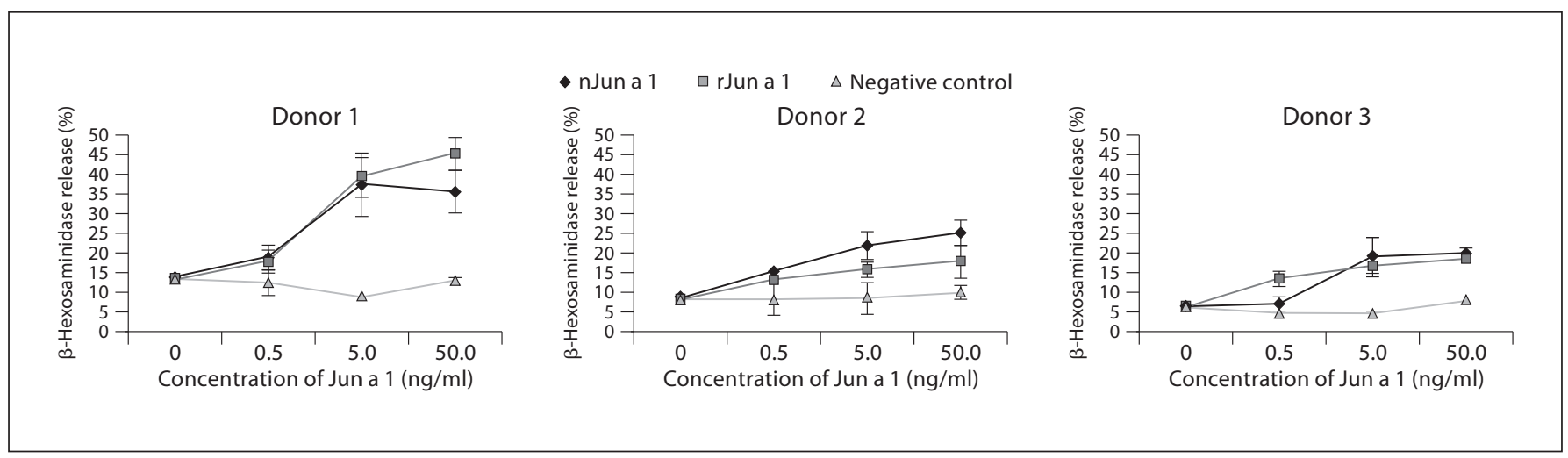

Fig. 10. Biological activity of nJun a 1 and rJun a 1. Induction of $\beta$-hexosaminidase release from RBL SX-38 cells sensitized with sera from mountain cedar-allergic patients (same three subjects as in fig. 7) and then stimulated with either purified nJun a 1 or rJun a 1 . The flow-through (FT) from affinity chromatography column (fig. 2a) served as negative control. ANOVA shows there is no significant between-subject (nJun a 1 versus rJun a 1) effect for donor $1(\mathrm{p}=0.799)$, for donor $2(\mathrm{p}=0.411)$ and for donor $3(\mathrm{p}=0.608)$.

and Jun a 1 . However, Jun a 1 differs structurally and chemically in several ways (for example, lack of $\mathrm{Ca}^{2+}$ binding) from microbial pectate lyases. The very low pectate lyase specific activity for nJun a 1 we observed (minimum of $0.2 \mathrm{mU} / \mathrm{mg}$ protein) in this study for relative to the pectate lyases activity $(20-2,000 \mathrm{U} / \mathrm{mg})$ of the plant pathogenic bacteria [24] may be related to the structural differences we found between nJun a 1 and the bacterial pectate lyases [5]. The current study also indicates that the nJun a 1 and rJun a 1 activities are 50 -fold lower than recombinant banana pectate lyase $(10.5 \mathrm{mU} / \mathrm{mg}$ protein) [10], and nearly 2 million-fold lower than the $350 \mathrm{U} / \mathrm{mg}$ protein reported for Japanese cedar pollen pectate lyase, Cry j 1 [11].

Cryj 1 and Jun a 1 have a greater than $80 \%$ amino acid sequence identity; however, without having the three-dimensional structure of Cry j 1, it is difficult to postulate the structural basis for the differences in pectate lyase activity between them. The extremely low pectate lyase activity also makes it somewhat difficult to attribute the toxicity of Jun a 1 expressed by tobacco leaves to pectate lyase activity and suggests that some plant pectate lyaselike proteins may have evolved a different biological function that is required for pollination and perhaps fruit 
maturation, but is toxic to other plant tissues. The different pectate lyase activity levels seen in these pollen proteins may not be relevant to this alternative function.

In order to begin to understand these unique structural features and functions of Jun a 1, mutants were created in two regions where Jun a 1 differed from the microbial pectate lyases. An $\mathrm{His}^{203}$ to Ala mutant was created in order to remove the histidine-aspartate bridge which is thought to transverse the putative substrate groove. Recombinant wild-type and $\mathrm{His}^{203}$ to Ala mutant of Jun a 1 were successfully expressed in $N$. benthamiana. However, the rate of substrate degradation by these proteins suggested less (rather than more) enzymatic activity in the His to Ala mutant, compared to wild-type Jun a 1. Further, we did not detect any difference in the onset or extent of plant necrosis between the wild type and the $\mathrm{Ala}^{203}$ to His mutant. We have not had an opportunity to compare the relative binding of human IgE antibodies between the mutant and wild-type rJun a 1. Again, it is interesting to speculate about the loss of the histidineaspartate bridge-reduced pectate lyase activity, but perhaps it promotes the binding of another substrate or enhances a different enzymatic activity. We do not yet have the crystal structure of this variant to confirm that the groove is changed and, if so, to what extent.

Truncation mutants were made by eliminating the first 38 amino acids, which were posited to form a loop structure that inhibited enzymatic activity, in this case by covering the vWiDH sequence [25]. No Jun a 1 protein was detected in plants inoculated with virus carrying the truncated sequence, indicating a deletion of the gene from the vector. Consistent with this finding, these plants did not develop any necrosis. The lack of Jun a 1 expression from $\mathrm{N}$-terminal truncation mutants may indicate that the truncated $\mathrm{N}$-terminus mutant of Jun a 1 is especially toxic to the cell, which favored the replication of vectors with a deleted payload. We have observed that toxic proteins (for example, TNFR death domain) expressed in the TMV vector result in swift elimination of the insert upon replication in plants.

The plant viral expression system described here is currently the only platform that we have found that can produce immunologically active recombinant allergen Jun a 1. In our hands, Jun a 1 has been difficult to express in bacteria in a soluble, correctly folded state. Baculovirus infection in insect cells has also been attempted, but the baculovirus-expressed Jun a 1 constructs were toxic to the insect cells, leading to cell death and very poor expression of Jun a 1. Limited experiments in fungal systems have not been encouraging. Recombinant mutant Jun a 1 can now be made in the TMV expression system in sufficient quantity and quality for structure/function studies.

TMV expression systems have been used to produce full, tetrameric IgG antibodies by ICON Genetics Inc. (Princeton, N.J., USA) [26], a number of vaccine candidates by Fraunhofer USA (Plymouth, Mich., USA) [e.g, $33,34]$ and scFv vaccines against lymphoma [16] by Large Scale Biology Inc. (Vacaville, Calif., USA) have reached clinical trials. Though we regard this system as useful primarily to explore Jun a 1 structure and function, the commercial development of other approved pharmaceutical proteins by the TMV system would open the possibility of producing safe and effective agents for specific immunotherapy against Jun a 1-driven allergic disease.

\section{Acknowledgements}

This work was supported by grants from the NIAID (R01 AI052428, R.M.G.; K08 AI055792, T.M.H.) and the NIEHS Center for Environmental Science (ES06676, R.M.G., T.M.H.).

\section{References}

1 Goetz DW, Goetz MA, Whisman BA: Mountain cedar allergens found in nonpollen tree parts. Ann Allergy Asthma Immunol 1995; 75:256-260.

2 Midoro-Horiuti T, Goldblum RM, Kurosky A, Goetz DW, Brooks EG: Isolation and characterization of the mountain cedar $(J u-$ niperus ashei) pollen major allergen, Jun a 1. J Allergy Clin Immunol 1999;104:608-612.
- 3 Midoro-Horiuti T, Goldblum RM, Kurosky A, Wood TG, Schein CH, Brooks EG: Molecular cloning of the mountain cedar (Juniperus ashei) pollen major allergen, Jun a 1. J Allergy Clin Immunol. 1999; 104(3 Pt 1):613617.

-4 Midoro-Horiuti T, Mathura V, Schein $\mathrm{CH}$, Braun W, Yu S, Watanabe M, et al: Major linear IgE epitopes of mountain cedar pollen allergen Jun a 1 map to the pectate lyase catalytic site. Mol Immunol 2003;40:555562 .
Czerwinski EW, Midoro-Horiuti T, White MA, Brooks EG, Goldblum RM: Crystal structure of Jun a 1, the major cedar pollen allergen from juniperus ashei, reveals a parallel beta-helical core. J Biol Chem 2005;280: 3740-3746.

-6 Moehnke MH, Midoro-Horiuti T, Goldblum RM, Kearney CM: The expression of a mountain cedar allergen comparing plantviral apoplastic and yeast expression systems. Biotechnol Lett 2008;30:1259-1264. 
7 Krebitz M, Wiedermann U, Essl D, Steinkellner H, Wagner B, Turpen TH, et al: Rapid production of the major birch pollen allergen Bet $\mathrm{v} 1$ in Nicotiana benthamiana plants and its immunological in vitro and in vivo characterization. FASEB J 2000;14:1279-1288.

$>8$ Krebitz M, Wagner B, Ferreira F, Peterbauer C, Campillo N, Witty M, et al: Plant-based heterologous expression of Mal d 2, a thaumatin-like protein and allergen of apple (Malus domestica), and its characterization as an antifungal protein. J Mol Biol 2003;329: 721-730.

$\checkmark 9$ Burtin D, Chabre H, Olagnier B, Didierlaurent A, Couret MN, Comeau D, et al: Production of native and modified recombinant Der p 1 molecules in tobacco plants. Clin Exp Allergy 2009;39:760-770.

10 Marin-Rodriguez MC, Smith DL, Manning K, Orchard J, Seymour GB: Pectate lyase gene expression and enzyme activity in ripening banana fruit. Plant Mol Biol 2003;51: 851-857.

-11 Taniguchi Y, Ono A, Sawatani M, Nanba M, Kohno K, Usui M, et al: Cry j I, a major allergen of japanese cedar pollen, has pectate lyase enzyme activity. Allergy 1995;50:9093.

-12 Herron SR, Benen JA, Scavetta RD, Visser J, Jurnak F: Structure and function of pectic enzymes: virulence factors of plant pathogens. Proc Natl Acad Sci USA 2000;97:87628769 .
13 Platts-Mills TA, Mueller GA, Wheatley LM: Future directions for allergen immunotherapy. J Allergy Clin Immunol 1998;102:335343.

14 Varshney S, Goldblum RM, Auton M, Kearney C, Watanabe M, Midoro-Horiuti T: Major mountain cedar allergen, Jun a 1 contains conformational as well as linear IgE epitopes. Mol Immunol 2007;44:2781-2785.

$>15$ Lindbo JA: High-efficiency protein expression in plants from agroinfection-compatible tobacco mosaic virus expression vectors. BMC Biotechnol 2007;7:52.

16 McCormick AA, Kumagai MH, Hanley K, Turpen TH, Hakim I, Grill LK, et al: Rapid production of specific vaccines for lymphoma by expression of the tumor-derived single-chain fv epitopes in tobacco plants. Proc Natl Acad Sci USA 1999;96:703-708.

17 Zheng Y, Mort A: Isolation and structural characterization of a novel oligosaccharide from the rhamnogalacturonan of Gossypium hirsutum L. Carbohydr Res 2008;343:10411049.

18 Naran R, Pierce ML, Mort AJ: Detection and identification of rhamnogalacturonan lyase activity in intercellular spaces of expanding cotton cotyledons. Plant J 2007;50:95-107.

19 Kinet JP: The crystal structure of the human high-affinity IgE receptor (Fc $\varepsilon$ RI $\alpha$ ). Ann Rev Immunol 1999;17:931-972.

20 Wiegand TW, Williams PB, Dreskin SC, Jouvin MH, Kinet JP, Tasset D: High-affinity oligonucleotide ligands to human IgE inhibit binding to Fc $\varepsilon$ receptor I. J Immunol 1996; 157:221-230.
21 Dastych J, Walczak-Drzewiecka A, Wyczolkowska J, Metcalfe DD: Murine mast cells exposed to mercuric chloride release granule-associated $\mathrm{N}$-acetyl- $\beta$-D-hexosaminidase and secrete IL-4 and TNF- $\alpha$. J Allergy Clin Immunol 1999;103:1108-1114.

22 Zhu FG, Gomi K, Marshall JS: Short-term and long-term cytokine release by mouse bone marrow mast cells and the differentiated KU-812 cell line are inhibited by brefeldin A. J Immunol 1998;161:2541-2551.

23 Zhang Z, Pierce ML, Mort AJ: Changes in homogalacturonans and enzymes degrading them during cotton cotyledon expansion. Phytochemistry 2007;68:1094-1103.

24 Tardy F, Nasser W, Robert-Baudouy J, Hugouvieux-Cotte-Pattat N: Comparative analysis of the five major Erwinia chrysanthemi pectate lyases: enzyme characteristics and potential inhibitors. J Bacteriol 1997; 179:2503-2511.

25 Kita N, Boyd CM, Garrett MR, Jurnak F, Keen NT: Differential effect of site-directed mutations in pelC on pectate lyase activity, plant tissue maceration, and elicitor activity. J Biol Chem 1996;271:26529-26535.

26 Giritch A, Marillonnet S, Engler C, van Eldik G, Botterman J, Klimyuk V, Gleba Y: Rapid high-yield expression of full-size IgG antibodies in plants coinfected with noncompeting viral vectors. Proc Natl Acad Sci USA 2006;103:14701-14706. 